\title{
Interaction between SARS-CoV helicase and a multifunctional cellular protein (Ddx5) revealed by yeast and mammalian cell two-hybrid systems
}

\author{
Jin-Yan Chen · Wan-Nan Chen • \\ Kwok-Man Vincent Poon • Bo-Jian Zheng • \\ Xu Lin · Yong-Xiang Wang $\cdot$ Yu-Mei Wen
}

Received: 3 August 2008/Accepted: 12 January 2009/Published online: 18 February 2009

(C) Springer-Verlag 2009

\begin{abstract}
To reveal the putative cellular factors involved in SARS coronavirus replication, the helicase (Hel, nsp13) of SARS coronavirus was used to screen the cDNA library of rat pulmonary epithelial cells using the yeast two-hybrid system. Positively interacting proteins were further tested using a mammalian cell hybrid system and co-immunoprecipitation in the human A549 cell line, which has been shown to support SARS coronavirus replication. Out of the seven positive clones observed by yeast two-hybrid assay, only the Ddx5 (Asp-Glu-Ala-Asp box polypeptide 5) protein showed specific interaction with SARS-CoV helicase. When expression of DdX5 was knocked down by small interfering RNA (siRNA), SARS coronavirus replication was significantly inhibited in fetal rhesus kidney (FRhK-4) cells. Since Ddx5 is a multifunctional protein that plays important roles in transcriptional regulation, its interaction with SARS coronavirus helicase provides interesting clues
\end{abstract}

Electronic supplementary material The online version of this article (doi:10.1007/s00705-009-0323-y) contains supplementary material, which is available to authorized users.

J.-Y. Chen · W.-N. Chen · X. Lin

Key Laboratory of Infection and Oncology,

Research Center of Molecular Medicine, Fujian Medical

University, 350004 Fuzhou, People's Republic of China

K.-M. V. Poon · B.-J. Zheng

Department of Microbiology, University of Hong Kong,

Hong Kong Special Administration Region,

People's Republic of China

Y.-X. Wang · Y.-M. Wen ( $\bowtie)$

Key Laboratory of Medical Molecular Virology,

Shanghai Medical College, Fudan University,

138 Yi Xue Yuan Road, 200032 Shanghai,

People's Republic of China

e-mail: ymwen@shmu.edu.cn for studying virus-host cell interactions in SARS-CoV infections.

The worldwide epidemic outbreak of severe acute respiratory syndrome (SARS) in 2003 was caused by a novel coronovirus $(\mathrm{CoV})$, designated SARS coronavirus (SARS$\mathrm{CoV}$ ). The $29.7-\mathrm{kb}$ positive-strand RNA genome of this virus has been sequenced [1-3]. In the $5^{\prime}$-region, the viral replicase gene (21221nt) encodes two large replicative polyproteins: pp1a (486 kDa) and pp1ab (790 kDa).The pp1a and pp1ab polyproteins are autoproteolytically processed by the main proteinase $\left(\mathrm{M}^{\mathrm{pro}}\right.$, or $\left.3 \mathrm{CL}^{\text {pro }}\right)$ to yield the functional components, including a single-strandedRNA-binding protein (nsp9), an RNA-dependent RNA polymerase (RdRp, nsp12), a short primer for nsp12, a superfamily-1-like helicase (Hel, nsp13) and a uridylatespecific endoribonuclease (nsp15) [4]. $\mathrm{M}^{\text {pro }}$, the RdRp and the Hel are all thought to be essential for the virus life cycle [5-7]. Coronavirus helicases are highly conserved, and the quaternary structure of SARS-CoV helicase has been predicted to possess two separate domains, i.e., the helicase domain (Hel) and a metal-binding domain (MBD) [8]. However, it remains unclear how the Hel and MBD are functionally related to one another and what implications this has for viral viability. Helicases have been targeted for the treatment of herpes simplex virus [9-11] and hepatitis C virus [12] infections, suggesting that the SARS-CoV helicase may also be a potential target in the search of new antiSARS drugs [9, 11-14]. As an example, bananins have been shown recently to inhibit the SARS-CoV helicase in vitro [15].

Various human proteins interacting with different genes of SARS-CoV have been identified or predicted [16]. 
However, host proteins interacting with the helicase have not yet been identified. In this study, we first employed the yeast two-hybrid system to address this issue, which was further verified by mammalian two-hybrid assay and in vivo co-immunoprecipitation to reveal the cellular cofactors interacting with SARS-CoV helicase.

SARS-CoV strain Urbani (AY278741) was used in this study. After the virus was propagated in Vero- 6 cells, viral RNA was extracted with TRIZOL (Invitrogen), and the full-length helicase gene, with a size of $1,800 \mathrm{bp}$, was amplified by one-step RT-PCR (Qiagen, Valencia, CA, USA). Using $\mathrm{Y} 2 \mathrm{H}$ helicaseF primer and $\mathrm{Y} 2 \mathrm{H}$ helicaseR primer (Supplementary Table 1), the Hel gene was amplified from the RT-PCR product and cloned into the yeast bait vector pGBKT7 (CLONTECH, NcoI and PstI) to generate construct pGBKT7-Hel. The construct was verified by sequencing. A rat lung cDNA library (Clontech, Japan) was used as the prey. The prey library was amplified according to the manual, and plasmids were prepared using the Qiagen Plasmid Mega extraction kit.

Expression of helicase was conducted by using the bait plasmid pGBKT7-Hel to transform the yeast strain AH109 through a small-scale transformation using lithium acetate. Transformants were lysed by urea/SDS ( $8 \mathrm{M}$ urea, 5\% SDS, 40 mM Tris-HCl, pH 6.8, 0.1 mM EDTA, $0.4 \mathrm{mg} / \mathrm{ml}$ bromophenol blue), the lysates were subjected to Western blotting, and the blots were probed with the GAL4 DNABD monoclonal antibody (Clontech). The colony-lift filter assay was used to measure the $\beta$-galactosidase activity of the reporter gene by helicase on Whatman $3 \mathrm{M}$ filters using $\mathrm{X}$-gal as substrate. pCL1 was used as a positive control, and pGBKT7-Lamin and pGBKT7 were used as negative controls. The successful expression of SARS-CoV helicase in yeast strain AH109 is shown in Fig. 1, and by colony-lift filter assay, the expression of SARS-CoV helicase in yeast strain AH109 was shown not to activate the MEL1 reporter gene (data not shown), which excluded self-activation.

Yeast two-hybrid screening was performed using a sequential transformation method as described in the MATCHMAKER GAL4 Two-Hybrid System 3 \& Libraries User Manual (Clontech). Briefly, the bait plasmid pGBKT7-Hel (TRP1) was first introduced into the haploid yeast strain AH109 using a small-scale transformation procedure, and the prey plasmids pACT2-X (LEU2) were sequentially introduced into AH109 [pGBKT7-Hel] using a large-scale procedure. The final AH109 transformants were re-suspended in YPDA medium and plated on SD/-Trp/Leu/-His/-Ade/X- $\alpha$-Gal selective plates (Quardruple dropout $=\mathrm{QDO}$, high-stringency plates for screening the expression of $A D E 2, H I S 3$, and MEL1) containing $1.25 \mathrm{mM} 3$-AT to inhibit background growth. The control plasmids used were pGBKT7-Lamin, pGBKT7-53, pGADT7-T, pGBKT7 and pACT2. One hundred thirty-three

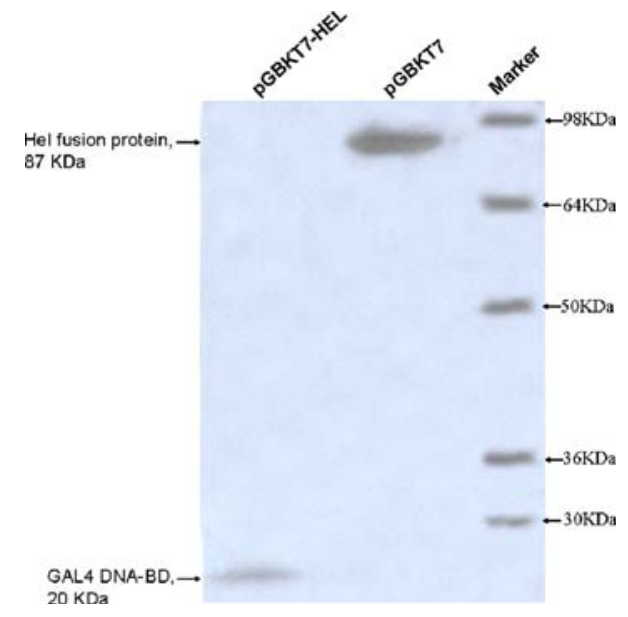

Fig. 1 Western blot analysis of SARS-CoV helicase in AH109 cells. pGBKT7-Hel was introduced by transformation into AH109, and the lysates of transformants were transferred to Western blots and probed with GAL4 DNA-BD monoclonal antibodies. The empty vector pGBKT7, encoding a GAL4 BD tag protein, is shown, with a molecular weight of around $20 \mathrm{kDa}$, while pGBKT7-Hel encoding a GAL4 BD-Hel fusion protein, is shown, with a molecular weight around $87 \mathrm{kDa}$

colonies that grew on the QDO plates were re-streaked on the selective plates in triplicate. Blue colonies were further assayed for $\beta$-galactosidase activity within $8 \mathrm{~h}$ on Whatman $3 \mathrm{M}$ filters using X-gal as substrate, and pCL1 (Clontech) was used as a positive control in the $\beta$-gal assay.

To isolate the bait and AD/library plasmids and sort colonies to eliminate duplicates, purified plasmid DNA extracted from the positive yeast colonies was introduced by transformation into $E$. coli strain $\mathrm{DH} 5 \alpha$, and the $\mathrm{AD} /$ library plasmids were digested separately with $A l u \mathrm{I}$ and $B g l \mathrm{II}$. To retest individual protein interactions in yeast, the $\mathrm{AD} /$ library plasmids were transformed into AH109 and the bait plasmid into Y187. Each $\beta$-galactosidase-negative AH109 transformant was mated with Y187 transformants to select for true positive diploids. Finally, the sequences of the inserts in the true positive $\mathrm{AD} /$ library plasmids were analyzed using the $3^{\prime}$ AD Sequencing Primer and T7 Sequencing Primer (Clontech). By colony sorting (AluI and BglII digestion), a total of 47 clones were selected among the 133 clones that grew on SD/QDO/X- $\alpha-\mathrm{Gal} / 3-\mathrm{AT}$ plates. Five out of the 47 clones showed autoactivation and were discarded. The other 42 clones were individually tested by mating with Y187 (pGBKT7-Hel) or Y187 (empty pGBKT7 vector). An interaction was considered to be a "true positive" only if it fulfilled the following criteria: (1) Diploids of the prey plasmid mating with pGBKT7-Hel grew on QDO plates. (2) Diploids of the prey plasmid mating with empty vector pGBKT7 did not grow on QDO plates. (3) The ORF of inserts matched the protein coding region(s) in BLASTP search. Only seven colonies, i.e., \#28, \#37, \#42, \#44, \#77, $\# 91$, and \#123 fulfilled these criteria (Table 1). 
Since post-translational processing in yeast may be different from that in mammalian cells, the mammalian twohybrid system was further used to verify the findings from the yeast two-hybrid assay. A549 cells (human, Caucasian, lung, carcinoma), which have been shown to support the growth of SARS-CoV, were used in mammalian two-hybrid studies followed by in vivo co-immunoprecipitation. The mammalian expression vector pBIND (Promega) and the coimmunoprecipitation vector pCMV-HA (Clontech) were used to generate constructs harboring the helicase gene of SARS-CoV. The two constructs were verified by sequencing and named pBIND-Hel and pCMV-HA-Hel, respectively. The primers used for cloning are listed in Supplementary Table 1.

Mammalian two-hybrid assays were performed following the instruction of the CheckMate ${ }^{\mathrm{TM}}$ Mammalian TwoHybrid System (Promega). The genes of interest, including those of \#28, \#37, \#42, \#44, \#77, \#91, and \#123, were amplified separately by PCR and cloned into pACT (Promega) to generate fusion proteins with the activation domain of VP16 (the primers used are listed in Supplementary Table 1). Co-transfection of A549 cells with the seven constructs (\#28, \#37, \#42, \#44, \#77, \#91, and \#123) along with the reporter plasmid pG5luc, which encodes firefly luciferase, was performed using Lipofectamine 2000 reagent (Invitrogen). Paired plasmids pBIND-Id and pACT-MyoD were used as positive controls, and the empty plasmids pBIND and pACT were used as negative controls. Each co-transfection was repeated five times. Firefly luciferase activity and Renilla luciferase activity were measured using the Dual-Luciferase ${ }^{\circledR}$ Reporter Assay System (Promega). Renilla luciferase activity was used to normalize the differences in transfection efficiencies. As showed in Table 2, by comparing the relative intracellular firefly luciferase activity of each co-transfected clone with its background control, among the seven clones that were previously positive in the yeast two-hybrid assay, only clone \#42, encoding Ddx5, was found to be positive in the mammalian two-hybrid assay. The relative intracellular firefly luciferase activity cotransfected with clone \#42, encoding Ddx5, was significantly higher than that of the corresponding background controls $(P<0.001)$. These results suggested that $\mathrm{SARS}-\mathrm{CoV}$ helicase could interact with Ddx5 in A549 cells.

To confirm that the protein interaction between SARSCoV helicase and Ddx5 occurred in vivo, co-immunoprecipitation was performed in cell culture. The Ddx5 gene was amplified (see Supplementary Table 1 for primers) and cloned into the plasmid pCMV-Myc (Clotech), and A549 cells were co-transfected with the construct pCMV-MycDdx5 and plasmid pCMV-HA-Hel. Cells were harvested and lysed $48 \mathrm{~h}$ post-transfection, and cell lysates were first precleared by treatment with Protein A-Agarose (Invitrogen), followed by precipitation with rabbit polyclonal anti-HA IgG (Sigma). The precipitated complexes were separated by sodium dodecyl sulphate-polyacrylamide gel electrophoresis (SDS-PAGE) and transferred onto PVDF membranes. The blots were first reacted with anti-c-Myc monoclonal antibodies (1:200, Clontech) as the primary antibodies and were subsequently detected using alkaline-phosphatase-conjugated secondary antibodies. Visualization of the immunoreactive proteins was shown by using CDP STAR reagents (Roche, Germany). As shown in Fig. 2a, the Myc-Ddx5 protein was detected by the anti-HA Ab, while the co-immunoprecipitation of Ddx5 with Hel from SARS-CoV was detected by anti-c-Myc Ab (Fig. 2b, lane 4). The results indicate that the Ddx5 protein interacted with helicase during immunoprecipitation. Importantly, no interactions were detected between the Ddx5 protein with lysates from the A549 cells co-transfected with the controls, which included pCMV-Myc plus HA-Hel (Fig. 2b, lane 2), pCMV-HA plus Myc-Ddx5 (Fig. 2b, lane 3), and pCMV-Myc plus pCMV-HA (both were empty vectors, Fig. 2b, lane 1).

To determine the effect of Ddx5 on SARS-CoV replication, the expression of $\operatorname{Ddx} 5$ was knocked down by siRNA targeting Ddx5 mRNA, and the viral load and titers were evaluated. Briefly, small interfering RNA (siRNA) oligonucleotides (Ddx5-1144, 5'-GGUUCUAAAUGAA UUCAAATT- $3^{\prime}$ ) targeting mRNA of Ddx5 (accession number NM_004396.2) and control unrelated siRNA (5'-UUCUCCGAACGUGUCACGUTT-3') were synthesized
Table 1 Hel-associated peptides identified by yeast two-hybrid screening

\begin{tabular}{lll}
\hline Clone & Potential Hel-associated proteins & Accession no. \\
\hline$\# 28$ & THAP domain containing 11 (Thap11) & XM_001075955 \\
$\# 37$ & $\begin{array}{l}\text { SWI/SNF-related, matrix-associated, actin-dependent regulator } \\
\text { of chromatin, subfamily b, member 1 (Smarcb1) }\end{array}$ & NM_001025728 \\
\#42 & ddx5 gene (Ddx5) & NM_001007613 \\
$\# 44$ & Breast carcinoma amplified sequence 2 (Bcas2) & XM_001064236 \\
$\# 77$ & Adenosine deaminase (Ada) & NM_130399 \\
$\# 91$ & Aspartyl-tRNA synthetase 2 (mitochondrial)(Dars2) & NM_001034143 \\
$\# 123$ & Actinin alpha 2 (Actn2) & XM_001061664 \\
\hline
\end{tabular}


Table 2 Relative intracellular firefly luciferase activity detected in A549 cells cotransfected with the seven potential genes

\begin{tabular}{|c|c|c|c|c|c|c|c|}
\hline \multirow[t]{2}{*}{ Plasmids for transfection } & \multicolumn{5}{|l|}{ Exp. } & \multirow[t]{2}{*}{$x \pm s$} & \multirow[t]{2}{*}{$P$ value } \\
\hline & 1 & 2 & 3 & 4 & 5 & & \\
\hline $\mathrm{pBIND}+\mathrm{pACT}$ & 1 & 1 & 1 & 1 & 1 & $1 \pm 0$ & \\
\hline pBIND-d + pACT-MyoD & $55.3 \#$ & 49.4 & 65.9 & 56.7 & 50.2 & $55.5 \pm 6.61$ & \\
\hline pBIND-el + pACT & 1.15 & 1.09 & 1.04 & 0.98 & 1.19 & $1.09 \pm 0.09$ & $>0.05^{*}$ \\
\hline pACT-28 + pBIND & 0.07 & 1.03 & 1.09 & 1.06 & 1 & $0.85 \pm 0.44$ & \\
\hline pACT-28 + pBIND-Hel & 1.01 & 1.05 & 1.66 & 1.12 & 1.09 & $1.19 \pm 0.27$ & $>0.05 * *$ \\
\hline pACT-37 + pBIND & 1.04 & 1.9 & 1.77 & 1.26 & 2.04 & $1.60 \pm 0.43$ & \\
\hline pACT-37 + pBIND-Hel & 1.14 & 2.21 & 2.23 & 2.24 & 2.58 & $2.08 \pm 0.55$ & $>0.05 * *$ \\
\hline pACT-42 + pBIND & 2.28 & 2.62 & 2.38 & 2.76 & 2.6 & $2.53 \pm 0.19$ & \\
\hline pACT-42 + pBIND-Hel & 4.72 & 5.8 & 6.76 & 4.24 & 5.78 & $5.46 \pm 0.99$ & $<0.001 * *$ \\
\hline pACT-44 + pBIND & 1.04 & 1.05 & 0.9 & 0.81 & 0.93 & $0.95 \pm 0.1$ & \\
\hline pACT-44 + pBIND-Hel & 1.12 & 1.55 & 1.09 & 1.13 & 1.02 & $1.18 \pm 0.21$ & $>0.05 * *$ \\
\hline pACT-77 + pBIND & 1.09 & 1.08 & 0.74 & 0.96 & 1.05 & $0.98 \pm 0.15$ & \\
\hline pACT-77 + pBIND-Hel & 1.11 & 1.18 & 0.98 & 1.32 & 1.17 & $1.15 \pm 0.12$ & $>0.05 * *$ \\
\hline pACT-91 + pBIND & 1.08 & 1.09 & 1.03 & 0.88 & 1.03 & $1.02 \pm 0.08$ & \\
\hline pACT-91 + pBIND-Hel & 1.16 & 1.59 & 1.19 & 1.03 & 1.12 & $1.22 \pm 0.22$ & $>0.05 * *$ \\
\hline pACT-123 + pBIND & 1.14 & 1.15 & 0.82 & 0.91 & 0.91 & $0.99 \pm 0.15$ & \\
\hline pACT-m123 + pBIND-Hel & 1.22 & 1.45 & 1.09 & 1.03 & 1.01 & $1.16 \pm 0.18$ & $>0.05 * *$ \\
\hline
\end{tabular}

\#The numbers are the measured relative intracellular firefly luciferase activity in each experiment. pACT and pBIND are the names of plasmids used for trasnfection, pBIND-Hel is the recombinant plasmid with the helicase insert, and pACT-m28, m37, m42, m44, m77, m91 and m123, represent recombinant plasmids encoding the clones with numbers relative to Table 1

* $t$ test between paired samples of pBIND-Hel cotransfected with pACT versus pBIND contransfected with pACT as the control

** $t$ tests between paired samples of each pACT recombinant clone (harboring-m28, m-37, m-42, m-44, m-77, m-91 and m-123 insert, respectively), either contransfected with pBIND or cotransfected with pBIND-Hel

A
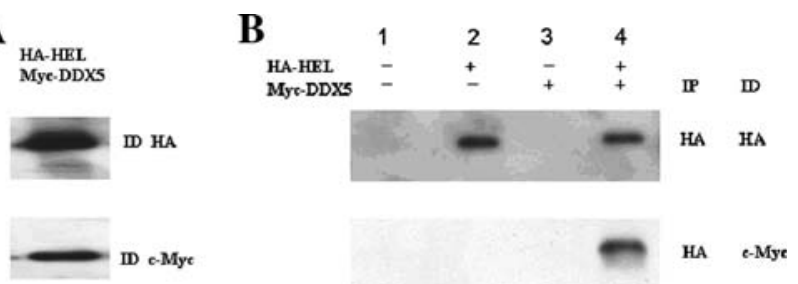

Fig. 2 In vivo co-immunoprecipitation of SARS-CoV helicase and cellular protein Ddx5 in A549 cells. a Immunoblotting of protein extracts from a cell line co-expressing HA-Hel with c-Myc-Ddx5, using anti-HA and anti-c-Myc antibodies. b Protein extracts from the cells were first subjected to overnight incubation with anti-HA IgG, and the co-precipitated proteins were detected with anti-c-Myc antibodies. IP and ID refer to the antibodies used for immunoprecipitation, and immunodetection, respectively. HA-Hel and/or MycDdx 5 proteins loaded into different lanes are indicated. Only lane 4 was loaded with Hel and Ddx5, which showed co-precipitation of both proteins

(GenePharma, Shanghai, China). Fetal rhesus kidney (FRhK-4) cells were transfected with $1.5 \mu \mathrm{M}$ siRNAs using LipofectamineTM RNAiMAX (Invitrogen, USA) in six-well plates, and the expression levels of Ddx5 were detected by western blot analysis (1: 100, anti-p68 RNA helicase, Santa Cruz) $72 \mathrm{~h}$ post-transfection. As shown in
Fig. 3a, the expression of Ddx 5 was dramatically reduced in FRhK-4 cells treated with Ddx5-specific siRNA, but not with control unrelated siRNA. After the FRhK-4 cells were transfected with siRNAs $(1.5 \mu \mathrm{M})$ for $16-18 \mathrm{~h}$ in 96 -well plates in duplicate, the transfected cells were infected with 100 TCID50 of SARS-CoV strain GZ-50 [17]. Supernatants were collected $72 \mathrm{~h}$ after viral infection to detect viral load by real-time RT-PCR and viral titers by back-titration as described previously [18-20]. The experiments were repeated twice. As shown in Fig. 3b, the viral RNA copy was inhibited by $70 \%$, and the viral titer of SARS-CoV strain GZ-50 was reduced by $97 \%$.The results showed that SARS coronavirus replication was significantly inhibited in FRhK-4 cells transfected with Ddx5-specific siRNA.

The roles of helicase of SARS-CoV in virus replication have been extensively studied [8, 21]. Ivanov et al. [22] have characterized the enzymatic activities of a recombinant form of the SARS-CoV helicase (nsp13) and reported that nsp13 has both RNA and DNA duplex unwinding activity. A recent study on intraviral protein-protein interactions revealed that nsp8 interacts with a number of replicase proteins, including nsp13 [16]. By reverse genetic studies, ORF1ab proteins were shown to be involved in 
A

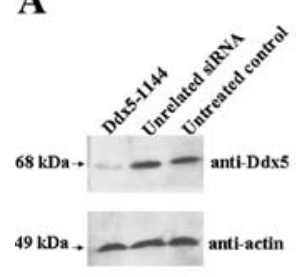

B

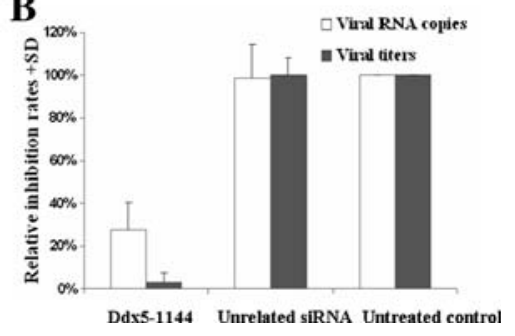

Fig. 3 Inhibition of siRNA on the expression of Ddx 5 protein (a) and replication of SARS-CoV (b) in FRhK-4 cells. a FRhK-4 cells were transfected with either Ddx5-specifc siRNA (Ddx5-1144, lane 1) or unrelated siRNA (lane 2), cells were disrupted $72 \mathrm{~h}$ post-transfection, and whole-cell lysates were examined by Western blotting using antiDdx5 antibody. b FRhK-4 cells were transfected with either Ddx51144 (group 1) or unrelated siRNA (group 2), and 16-18 h after transfection, the cells were infected with 100 TCID50 of SARS-CoV strain GZ-50. Supernatant was collected $72 \mathrm{~h}$ post-infection to measure viral load by real-time RT-PCR and viral titers by backtitration. The relative inhibition rate was compared to those for cells transfected with control siRNA and untreated cells

cellular signaling and modification of cellular gene expression, which might be related to viral virulence [23]. Since SARS-CoV replication is a highly complex process, interactions between helicase and cellular proteins, such as transcription factors, regulatory molecules and proteins controlling cell viability and proliferation, could be involved. In this study, we first used the yeast two-hybrid system to screen possible cellular proteins interacting with SARS-CoV helicase [24]. Aside from the advantage of the yeast two-hybrid system that it can screen a large number of protein-protein interactions in an intracellular setting, because enzymes are used as reporters that can amplify the signal, the yeast two-hybrid system is highly sensitive and can detect weak protein-protein interactions. On the other hand, since some proteins may have intrinsic transcriptional activation activity or are inherently sticky ("promiscuous interactors"), false positive results are commonly observed. Methods for eliminating or obviating this defect have been developed, and thus, the yeast strain AH109 used in this study contains three selective markers: $A D E 2, H I S 3$ and $M E L 1$ for higher stringency in screening and assay. Therefore, in this study only seven positive clones were obtained that needed to be verified.

By two-hybrid assay in mammalian cells and co-immunoprecipitation, Ddx 5 from A549 cells was shown to interact specifically and directly with the SARS-CoV helicase protein. It has been shown that SARS-CoV can replicate in the human-lung-derived A549 cell line [25], and we believe that this finding has important implications. Ddx 5 is a prototypical member of the DEAD box family of RNA helicases, which was first identified by cross-reaction with a monoclonal antibody [26, 27]. Ddx5 was one of the first proteins showing RNA helicase activity in vitro [28], and Ddx5 (p68) has been shown to be involved in many biological events related to RNA structure, such as transcription [29], premRNA processing, RNA degradation, RNA export, ribosome assembly and translation [30]. In addition, increasing evidence has indicated that Ddx5 (p68) interacts with many transcription factors, including several transcriptional coactivators and co-repressors. It interacts with and acts as a transcriptional coactivator for the nuclear receptor estrogen receptor alpha $(E R \alpha)[31]$. A recent report has suggested that p68 acts as a potent coactivator for the tumor suppressor $\mathrm{p} 53$, a latent transcription factor that is induced and deactivated in response to stresses, such as DNA damage, and induces transcription of genes involved in cell cycle arrest and apoptosis[32]. Ddx5 also interacts with many other transcription factors including SMAD3 [33] and MyoD. In addition, Ddx 5 also associates with RNA coactivator SRA [34], p300, CREB-binding protein (CBP) [35], RNA polymerase II and HDAC1 [36]. All of these data indicate that Ddx5 (p68) is involved in many cellular processes.

To our knowledge, this is the first report to show that SARS-CoV helicase can interact directly with multifunctional protein Ddx5 in cell culture and that inhibition of Ddx5 results in the suppression of viral replication. We speculate that Ddx5 may act as a coactivator by direct binding to the SARS-CoV helicase, resulting in enhanced viral genome transcription and virus proliferation. In addition, in view of the important functions of Ddx5, the cellular effects exerted by the complex formed between SARS helicase and Ddx5 needs further study.

Acknowledgment This work was supported by Sino-German collaborative grant GZ230 (202/3).

\section{References}

1. Ksiazek TG, Erdman D, Goldsmith CS, Zaki SR, Peret T, Emery S, Tong S, Urbani C, Comer JA et al (2003) A novel coronavirus associated with severe acute respiratory syndrome. N Engl J Med 348:1953-1966

2. Peiris JS, Chu CM, Cheng VC, Chan KS, Hung IF, Poon LL, Law KI, Tang BS, Hon TY et al (2003) Clinical progression and viral load in a community outbreak of coronavirus-associated SARS pneumonia: a prospective study. Lancet 361:1767-1772

3. Peiris JS, Yuen KY, Osterhaus AD, Stohr K (2003) The severe acute respiratory syndrome. N Engl J Med 349:2431-2441

4. Marra MA, Jones SJ, Astell CR, Holt RA, Brooks-Wilson A, Butterfield YS, Khattra J, Asano JK, Barber SA et al (2003) The genome sequence of the SARS-associated coronavirus. Science 300:1399-1404

5. Lai MM, Cavanagh D (1997) The molecular biology of coronaviruses. Adv Virus Res 48:1-100

6. Van Dinten LC, van Tol H, Gorbalenya AE, Snijder EJ (2000) The predicted metal-binding region of the Arterivirus Helicase protein is involved in subgenomic mRNA synthesis, genome replication, and virion biogenesis. J Virol 74:5213-5223

7. Kadare G, Haenni AL (1997) Virus-encoded RNA helicases. J Virol 71:2583-2590 
8. Andrea B, Ottavia S, Vincenzo V, Filippo P, Luisa B, Huang JD, Tanner JA, Neri N (2006) Tertiary structure prediction of SARS coronavirus helicase. Biochem Biophys Res Commun 343:11011104

9. Kleymann G, Fischer R, Betz UA, Hendrix M, Bender W, Schneider U, Handke G, Eckenberg P, Hewlett G et al (2002) New helicase-primase inhibitors as drug candidates for the treatment of herpes simplex disease. Nat Med 8:392-398

10. Kleymann G (2003) Novel agents and strategies to treat herpes simplex virus infections. Exp Opin Investig Drugs 12:165-183

11. Crute JJ, Grygon CA, Hargrave KD, Simoneau B, Faucher AM, Bolger G, Kibler P, Liuzzi M, Cordingley MG (2002) Herpes simplex virus helicase-primase inhibitors are active in animal models of human disease. Nat Med 8(4):386-391

12. Borowski P, Schalinski S, Schmitz H (2002) Nucleotide triphosphatase/helicase of hepatitis $\mathrm{C}$ virus as a target for antiviral therapy. Antiviral Res 55(3):397-412

13. Tanner JA, Watt RM, Chai YB, Lu LY, Lin MC, Peiris JSM, Poon LLM, Kung HF, Huang JD (2003) The severe acute respiratory syndrome (SARS) coronavirus NTP/helicase belongs to a distinct class of $5^{\prime}$ to $3^{\prime}$ viral helicase. J Biol Chem 278:39578-39582

14. Kao RY, Tsui WH, Lee TS, Tanner JA, Watt RM, Huang JD, Hu L, Chen G, Chen Z et al (2004) Identification of novel smallmolecule inhibitors of severe acute respiratory syndromeassociated coronavirus by chemical genetics. J Biol Chem 11:1293-1299

15. Andreas JK (2005) Synthesis of novel test compounds for antiviral chemotherapy of severe acute respiratory syndrome (SARS). Curr Med Chem 12:2095-2162

16. Von Albrecht B, Carola T, Jeremy CS, Rainer P, Caroline CF, Ralf Z, Rhonda R, Ralph B, Jürgen H (2007) Analysis of intraviral protein-protein interactions of the SARS coronavirus ORFeome. PLoS ONE 2(5):e459

17. Zhong NS, Zheng BJ, Li YM, Poon LLM, Xie ZH, Li PH, Tan SY, Chang Q, Xie JP, Liu XQ, Xu J, Li DX, Yuen KY, Peiris JSM, Guan Y (2003) Epidemiology and cause of severe acute respiratory syndrome (SARS) in Guangdong, People's Republic of China, in February, 2003. Lancet 362:1353-1358

18. He ML, Zheng B, Peiris JSM, Lin MCM, Peng Y, Poon LLM, Yuen KY, Kung HF, Guan Y (2003) Inhibition of SARS-associated coronavirus infection and replication by RNA interference. JAMA 290:2665-2666

19. Zheng BJ, Guan Y, Tang QQ, Cheng D, Xie FY, He ML, Chan KW, Wong KL, Lader E, Woodle MC, Lu PY, Li BJ, Zhong NS (2004) Prophylactic and therapeutic effects of small interfering RNA targeting SARS-coronavirus. Antivir Ther 9:365-374

20. Zheng BJ, Guan Y, He ML, Sun HZ, Du LY, Zheng Y, Wong KL, Chen H, Chen Y, Lu L, Tanner JA, Watt RM, Niccolai N, Bernini A, Spiga O, Woo PCY, Kung HF, Yuen KY, Huang JD (2005) Synthetic peptides outside the spike protein heptad repeat regions as potent inhibitors of SARS-associated coronavirus. Antivir Ther 10:393-403

21. Marcin H, Krystian E, Marcinvon G, Leszek R, Ewa B, Tomasz G, Tomasz S, Andrzej K (2006) Three dimensional model of severe acute respiratory syndrome coronavirus helicase ATPase catalytic domain and molecular design of severe acute respiratory syndrome coronavirus helicase inhibitors. J Comput Aided Mol Des 20:305-319

22. Thiel V, Ivanov KA, Putics A (2003) Mechanisms and enzymes involved in SARS coronavirus genome expression. J Gen Virol 84:2305-2315

23. Graham RL, Sparks JS, Eckerle LD, Sims AC, Denison MR (2008) SARS coronavirus replicase proteins in pathogenesis. Virus Res 133:88-100

24. Fields S, Song OK (1989) A novel genetic system to detect protein-protein interactions. Nature 340:245-246

25. Yen YT, Liao F, Hsiao CH, Kao CL, Chen YC, Wu-Hsieh BA (2006) Modeling the early events of severe acute respiratory syndrome coronavirus infection in vitro. J Virol 80:2684-2693

26. Crawford L, Leppard K, Lane D, Harlow E (1982) Cellular proteins reactive with monoclonal antibodies directed against simian virus 40 T-antigen. J Virol 42:612-620

27. Lane DP, Hoeffler WK (1980) SV40 large T shares an antigenic determinant with a cellular protein of molecular weight 68,000 . Nature 288:167-170

28. Hirling H, Scheffner M, Restle T, Stahl H (1989) RNA helicase activity associated with the human p68 protein. Nature 339:562564

29. Ford MJ, Anton IA, Lane DP (1988) Nuclear protein with sequence homology to translation initiation factore eIF-4A. Nature 332:736-738

30. Tanner NK, Linder P (2001) DExD/H box RNA helicases: from generic motors to specific dissociation functions. Mol Cell 8:251262

31. Endoh H, Maruyama K, Masuhiro Y, Kobayashi Y, Goto M, Tai H, Yanagisawa J, Metzger D, Hashimoto S, Kato S (1999) Purification and identification of p68 RNA helicase acting as a transcriptional coactivator specific for the activation function 1 of human estrogen receptor $\alpha$. Mol Cell Biol 19:5363-5372

32. Bates GJ, Nicol SM, Wilson BJ, Jacobs AM, Bourdon JC, Wardrop J, Gregory DJ, Lane DP, Perkins ND, Fuller-Pace FV (2005) The DEAD box protein p68:a novel transcriptional coactivator of the p53 tumour suppressor. EMBO J 24:543-553

33. Warner DR, Bhattacherjee V, Yin X, Singh S, Mukhopadhyay P, Pisano MM, Greene RM (2004) Functional interaction between Smad, CREB binding protein, and p68 RNA helicase. Biochem Biophys Res Commun 324:70-76

34. Caretti G, Schiltz RL, Dilworth FJ, Di Padova M, Zhao P, Ogryzko V, Fuller-Pace FV, Hoffman EP, Tapscott SJ, Sartorelli V (2006) The RNA helicase p68/p72 and noncoding RNA SRA are coregulators of MyoD and skeletal muscle differentiation. Dev Cell 11:547-560

35. Rossow KL, Janknecht R (2003) Synergism between p68 RNA helicase and the transcriptional coactivators CBP and $\mathrm{p} 300$. Oncogene 22:151-156

36. Wilson BJ, Bates GJ, Nicol SM, Gregory DJ, Perkins ND, FullerPace FV (2004) The p68 and p72 DEAD box RNA helicases interact with HDAC1 and repress transcription in a promoterspecific manner. BMC Mol Biol 5:11 